\title{
Multisite Exchange-Enhanced Barocaloric Response in $\mathrm{Mn}_{3} \mathrm{NiN}$
}

\author{
David Boldrin, ${ }^{1,}$ Eduardo Mendive-Tapia, ${ }^{2}$ Jan Zemen, ${ }^{3}$ Julie B. Staunton, ${ }^{2}$ Thomas Hansen, ${ }^{4}$ Araceli Aznar, ${ }^{5}$ \\ Josep-Lluís Tamarit, ${ }^{5}$ Maria Barrio, ${ }^{5}$ Pol Lloveras, ${ }^{5}$ Jiyeob Kim, ${ }^{6}$ Xavier Moya, ${ }^{6}$ and Lesley F. Cohen ${ }^{1}$ \\ ${ }^{1}$ Department of Physics, Blackett Laboratory, Imperial College London, \\ London SW7 2AZ, United Kingdom \\ ${ }^{2}$ Department of Physics, University of Warwick, Coventry CV4 7AL, United Kingdom \\ ${ }^{3}$ Faculty of Electrical Engineering, Czech Technical University in Prague, \\ Techniká 2, Prague 166 27, Czech Republic \\ ${ }^{4}$ Institut Laue-Langevin, CS 20156, 38042 Grenoble, Cedex 9, France \\ ${ }^{5}$ Departament de Física, EEBE, Campus Diagonal-Besòs and Barcelona Research Center in Multiscale \\ Science and Engineering, Universitat Politècnica de Catalunya, Eduard Maristany, \\ 10-14, 08019 Barcelona, Catalonia, Spain \\ ${ }^{6}$ Department of Materials Science, University of Cambridge, Cambridge CB3 OFS, United Kingdom
}

(Received 12 June 2018; revised manuscript received 28 September 2018; published 28 November 2018)

\begin{abstract}
We study the barocaloric effect (BCE) in the geometrically frustrated antiferromagnet $\mathrm{Mn}_{3} \mathrm{NiN}$ across the Néel transition temperature. Experimentally, we find a larger barocaloric entropy change by a factor of 1.6 than that recently discovered in the isostructural antiperovskite $\mathrm{Mn}_{3} \mathrm{GaN}$ despite significantly greater magnetovolume coupling in $\mathrm{Mn}_{3} \mathrm{GaN}$. By fitting experimental data to theory, we show that the larger BCE of $\mathrm{Mn}_{3} \mathrm{NiN}$ originates from multisite exchange interactions amongst the local Mn magnetic moments and their coupling with itinerant electron spins. Using this framework, we discuss the route to maximize the $\mathrm{BCE}$ in the wider $\mathrm{Mn}_{3} A \mathrm{~N}$ family.
\end{abstract}

DOI: 10.1103/PhysRevX.8.041035

Subject Areas: Condensed Matter Physics

\section{INTRODUCTION}

The emerging field of solid-state caloric cooling offers opportunities for greater energy-efficient refrigeration without the need for environmentally harmful chemicals. Magnetocaloric properties are by far the most studied, whereas mechanocalorics including baro- and elastocalorics are only beginning to gain prominence [1-3]. In magnetocalorics, the largest effects are found in materials with contributions from both localized and itinerant magnetic moments, so called mixed magnetism with competing exchange interactions. In the intermetallics $R \mathrm{Co}_{2}$ $\left(R=\right.$ rare earth) [4], $\mathrm{Mn}-\mathrm{Fe}(\mathrm{P}, \mathrm{Si})$ [2], and $\mathrm{La}(\mathrm{Fe}, \mathrm{Si})_{13}$ $[5,6]$ mixing of magnetic contributions and a strong coupling to the crystal lattice results in first-order transition behavior while maintaining large magnetization and a high transition temperature. These properties also lend themselves to attractive barocaloric effects (BCEs), and this complementary behavior has guided initial research in this

\footnotetext{
*Corresponding author. d.boldrin@imperial.ac.uk

Published by the American Physical Society under the terms of the Creative Commons Attribution 4.0 International license. Further distribution of this work must maintain attribution to the author(s) and the published article's title, journal citation, and DOI.
}

field [3,7-11]. Recent efforts have also focused on qualitatively different material families such as ferrielectric inorganic salts [12], superionic conductors [13], and hybrid inorganic organic materials [14]. While impressive BCEs are achieved in these materials, low density of the materials, poor thermal conductivity, and long-term stability are issues that may limit their applicability. An alternative and promising family of magnetic systems which we explore here is geometrically frustrated antiferromagnets (AFMs) [15]. The most prominent example is the metallic alloy $\mathrm{Mn}_{3} \mathrm{GaN}$ with giant BCEs and a large volume change at the transition where the BCE is thought to be enhanced due to the stabilization of the local moment in the noncollinear AFM phase. The authors of Ref. [15] propose that the frustrated exchange interactions in the noncollinear AFM phase lead to a relative insensitivity of the transition temperature to pressure in comparison with other magnetic barocaloric materials, and it is this feature combined with the large volume change that underlies the impressive performance of the material. Thus, the combination of geometric frustration of exchange between local $\mathrm{Mn}$ moments and itinerant electrons, akin to the local and itinerant character of the optimum magnetocalorics, may provide a fruitful playground through which to explore new barocaloric materials.

The $\mathrm{Mn}_{3} A \mathrm{~N}$ family displays a number of unusual properties, such as anomalous coefficient of resistivity 
[16], negative thermal expansion [17], and piezomagnetism [18-20]. The origin of these properties lies in a combination of electronic features: (i) large local magnetic moments due to a half-filled $d$ state, (ii) a noncollinear AFM order born from frustrated $\mathrm{Mn}-\mathrm{Mn}$ magnetic interactions, and (iii) the mixing of itinerant and localized spin degrees of freedom and the self-sustaining interactions that occur between them $[15,18,21]$. The two noncollinear magnetic structures commonly found in the $\mathrm{Mn}_{3} A \mathrm{~N}$ family are shown in Figs. 1(a) and 1(b) and are hereafter termed $\Gamma^{4 g}$ and $\Gamma^{5 g}$, respectively. These structures are similar to

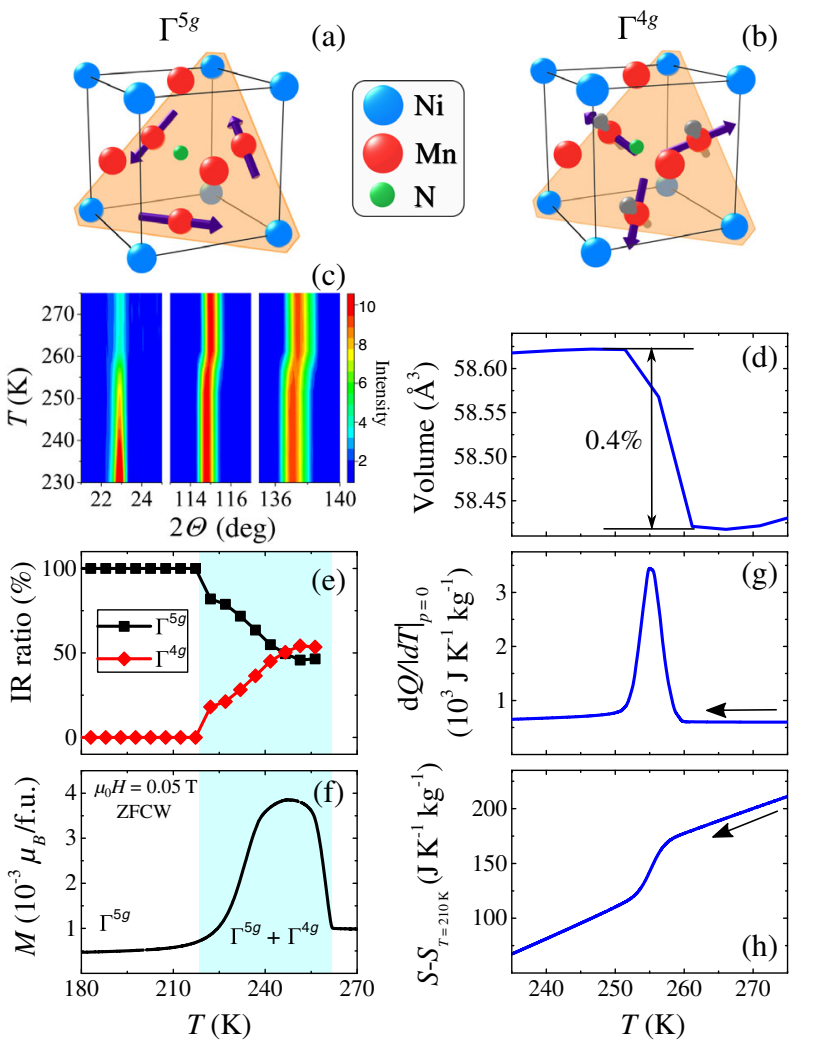

FIG. 1. (a) $\Gamma^{5 g}$ and (b) $\Gamma^{4 g}$ magnetic structures of $\mathrm{Mn}_{3} \mathrm{NiN}$. $\Gamma^{5 g}$ is a fully compensated antiferromagnetic structure stable at low temperatures. $\Gamma^{4 g}$ is also an antiferromagnetic structure (purple arrows) but has a symmetry-allowed ferromagnetic component (gray arrows) causing canting of the spins out of the (111) plane. (c) Thermodiffractogram measured using neutron powder diffraction $(\lambda=1.544 \AA$ ). (d) Unit-cell volume as a function of the temperature refined from the neutron-diffraction data. (e) The refined contributions of the $\Gamma^{5 g}$ and $\Gamma^{4 g}$ representations to the magnetic structure. (f) Bulk magnetometry data collected on the same sample under an applied field $\mu_{0} H=0.05 \mathrm{~T}$ after zerofield cooling. The blue highlighted region indicates the enlarged moment associated with the ferromagnetic contribution allowed in the $\Gamma^{4 g}$ representation. The transition region is $(\mathrm{g})$ temperaturedependent heat flow at ambient pressure $\left.[(d Q) /(d T)]\right|_{p=0}$, which is equal to the heat capacity outside the transition region and includes contributions that arise from latent heat within, and (h) is the total entropy change calculated from $(\mathrm{g})$ for the same sample on cooling. that which underlies the large anomalous Hall effect in the AFM $\mathrm{Mn}_{3} \mathrm{Sn}$, the only difference being the sign of the chirality [22]. The intrinsic frustration that leads to these noncollinear structures, combined with the energetic stability of their cubic lattice with respect to structural perturbations of lower symmetry, results in large relative volume changes $\Delta \omega_{T_{N}}$ at the first-order Néel transition temperature $T_{N}$, and a relative insensitivity of $T_{N}$ to pressure $p$. From these properties, a BCE figure of merit (FOM) $\Delta \omega_{T_{N}}\left|\left[\left(d T_{N}\right) /(d p)\right]\right|^{-1}[15]$ is proposed that allows comparisons to be made between material families. Here we measure the $\mathrm{BCE}$ in the closely related $\mathrm{Mn}_{3} \mathrm{NiN}$ $\left(T_{N}=262 \mathrm{~K}\right)$. Although intuitively it might be expected that larger volume changes at the transition will lead to a larger BCE, the entropy change under hydrostatic pressure in $\mathrm{Mn}_{3} \mathrm{NiN}$, in fact, outperforms $\mathrm{Mn}_{3} \mathrm{GaN}$ despite a significantly smaller volume change. We find that the enhanced barocaloric properties in $\mathrm{Mn}_{3} \mathrm{NiN}$ can be explained in terms of a combination of multisite exchange and magnetovolume coupling. This insight has implications for the ongoing search for improved barocaloric materials going forward.

\section{AMBIENT PRESSURE MEASUREMENTS}

We first discuss measurements performed on $\mathrm{Mn}_{3} \mathrm{NiN}$ at ambient pressure. The sample is prepared using a standard solid-state synthesis technique, as described previously [23]. Neutron-powder-diffraction data are collected on the D20 diffractometer ( $\lambda=1.544 \AA)$ at ILL, France [24]. Rietveld refinement of the data confirms that the sample is the antiperovskite $\mathrm{Mn}_{3} \mathrm{NiN}$ phase with only a minor $\mathrm{MnO}$ impurity (see Fig. S1 in the Supplemental Material [25]). The refined lattice parameter at room temperature is 3.880 75(3) A, in excellent agreement with the literature [26], and all atomic sites are refined to within $98 \%$ occupation, thus, confirming that the sample is close to stoichiometric with a fully occupied $\mathrm{N}$ site. The $\mathrm{N}$ site occupation is particularly noteworthy, as nitrogen deficiency is reported to occur in manganese nitride compounds [27].

The temperature dependence of the neutron-diffraction data is summarized in Fig. 1(c). At $T_{N}$, a clear shift in the nuclear Bragg peaks indicates a significant change in lattice parameters concomitant with the appearance of magnetic Bragg peaks. The refined lattice parameter across the transition reveals a volume change of $\Delta \omega_{T_{N}}=0.4 \%$ [Fig. 1(d)], which is similar to that found in previous studies on this material $[27,28]$. The magnetic Bragg peaks are centered on the nuclear peaks, thus, confirming a $k=0$ magnetic propagation vector. The temperature dependence of the magnetic structure is similar to that found previously [26]. At $T_{N}$, the magnetic moments are rotated within the (111) plane roughly between the $\Gamma^{4 g}$ and $\Gamma^{5 g}$ structures, and upon further cooling, they rotate towards the $\Gamma^{5 g}$ structure until approximately $220 \mathrm{~K}$ when this rotation is complete 
[Fig. 1(e)]. This temperature window coincides with an enlarged magnetization evidenced from the bulk magnetometry data [Fig. 1(f)], which can be explained by the symmetry-allowed ferromagnetic component of the $\Gamma^{4 g}$ structure [Fig. 1(b)] [26].

Evidence of the first-order character of the transition is found in the latent heat $\left(Q_{t} \sim T_{N} \Delta S_{t}\right)$ from the heat-flow data shown in Fig. 1(g). The total entropy change in zero pressure $S-S_{210 \mathrm{~K}}$ calculated from the same data as $S-S_{210 \mathrm{~K}}=\int_{210 \mathrm{~K}}^{T}\left\{1 / T[(d Q) /(d T)]_{p=0}\right\} d T$ is presented in Fig. 1(h). We find the entropy change across $T_{N},\left|\Delta S_{t}\right|$, at zero pressure is $43 \mathrm{~J} \mathrm{~K}^{-1} \mathrm{~kg}^{-1}$, approximately double the value found in $\mathrm{Mn}_{3} \mathrm{GaN}$ [15].

\section{PRESSURE-DEPENDENT AND BAROCALORIC EFFECT MEASUREMENTS}

We now turn to measurements performed under pressure (see the experimental details in the Supplemental Material [25]). The vibrating sample magnetometry (VSM) data of Fig. 2(a) show that $T_{N}$ shifts to lower $T$ with increased pressure. Moreover, in zero pressure the thermal hysteresis is $8 \mathrm{~K}$, while under a pressure of $0.61 \mathrm{GPa}$, the hysteresis is reduced to $7 \mathrm{~K}$ and the transition is broadened noticeably. As shown in Fig. 2(b), the pressure sensitivity of $T_{N}$ ranges between $-13 \pm 1$ and $-14 \pm 1 \mathrm{~K} \mathrm{GPa}^{-1}$, as determined by either high-pressure calorimetry (HC, explained below) or VSM, showing that they are in excellent agreement.

The results under pressure allow us to make a direct comparison with available data on the $\mathrm{Mn}_{3} \mathrm{GaN}$ antiperovskite [15]. First, $\Delta \omega_{T_{N}}$ at the transition of $0.4 \%$ is roughly half the value of that in the Ga system [27]. However, the sensitivity of $T_{N}$ with pressure
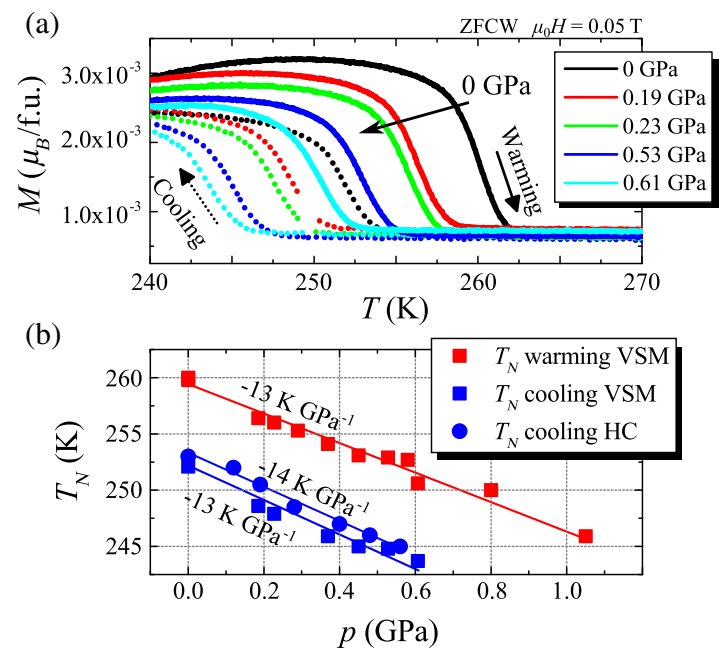

FIG. 2. (a) Magnetization under an applied field of $\mu_{0} H=$ $0.05 \mathrm{~T}$ as a function of the temperature and measured at different pressures after zero-field cooling. (b) Pressure dependence of $T_{N}$ taken from the derivative of the $M(T)$ VSM data and the peak of the HC data. Solid lines are linear fits to the data. $\left|\left[\left(d T_{N}\right) /(d p)\right]\right|$ of $13.5 \mathrm{~K} \mathrm{GPa}^{-1}$ (taken from the average of the measured values) is approximately 5 times smaller. If the magnetovolume coupling were the primary underlying mechanism for first-order character, one would expect $\Delta \omega_{T_{N}}$ and $\left|\left[\left(d T_{N}\right) /(d p)\right]\right|$ to scale linearly, given the chemical and magnetic similarities of the two systems and that both parameters are heavily dependent on the strength of the magnetovolume coupling. By combining these values, we find the FOM $\Delta \omega_{T_{N}}\left|\left[\left(d T_{N}\right) /(d p)\right]\right|^{-1}$ [15] in $\mathrm{Mn}_{3} \mathrm{NiN}$ is $0.03 \% \mathrm{GPa} \mathrm{K}^{-1}$, more than double that of $\mathrm{Mn}_{3} \mathrm{GaN}$ and several other known barocaloric systems [15].

We determine the $\mathrm{BCE}$ by quasidirect caloric measurements under pressure (see the experimental details in the Supplemental Material [25]). Temperature-dependent heatflow measurements under pressure $\left.[(d Q) /(d T)]\right|_{p}$ recorded on cooling [Fig. 3(a)] show a sharp peak at $T_{N}$ that shifts to lower temperatures with applied pressure. Figure 3(b) shows that the entropy change under applied pressures decreases, falling to a value upon cooling of $34 \mathrm{~J} \mathrm{~K}^{-1} \mathrm{~kg}^{-1}$ in $0.56 \mathrm{GPa}$. This decrease is not due to additional entropy
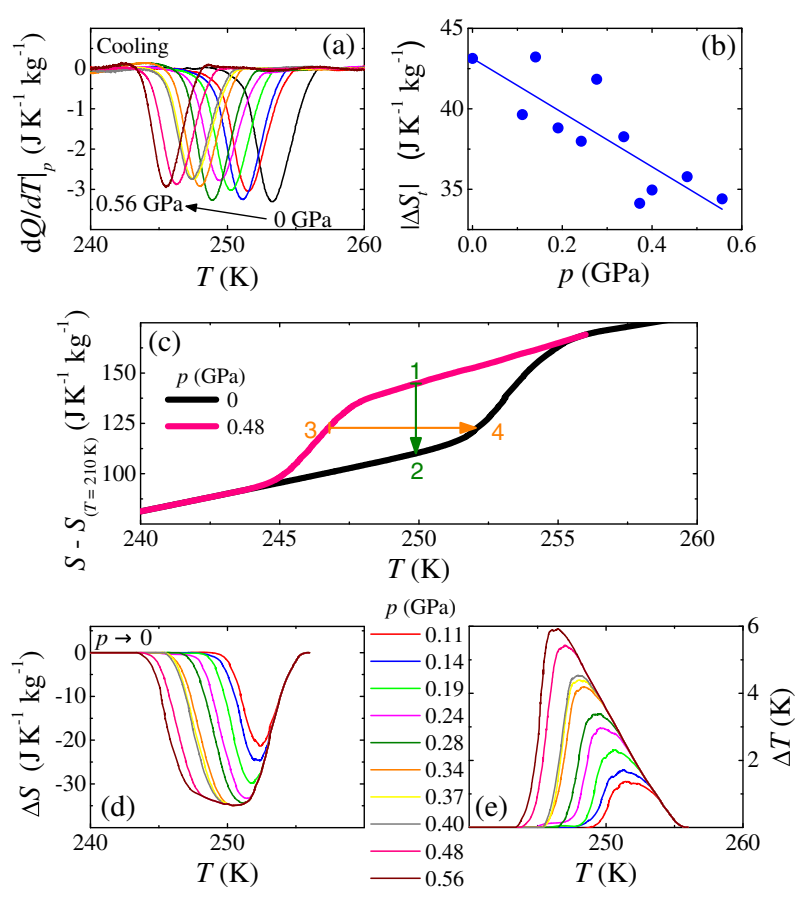

FIG. 3. (a) Temperature-dependent heat flow under pressure $\left.[(d Q) /(d T)]\right|_{p}$ on cooling through the transition at different values of increasing pressure $p$ after baseline subtraction. The full legend is the same as in (d) and (e). (b) Entropy changes cooling through $T_{N}$ obtained from the data in (a). (c) Isobaric entropy curves $S-S_{210 \text { K }}$ on cooling at 0- and 0.48-GPa applied pressure. The green arrow from 1 to 2 and orange arrow from 3 to 4 indicate the isothermal entropy and adiabatic temperature changes, respectively, upon removal of pressure. (d),(e) Isothermal entropy and adiabatic temperature changes obtained from the data in (a) as explained in (c). 
changes associated with the thermal expansion on either side of the transition [12], as these are negligible [Fig. 1(d)]. One explanation could be that the transition broadens (becomes more weakly first order) with increased pressure, which is consistent with the shape of the $M(T)$ curve in the transition region [Fig. 2(a)]. The reduction in magnetization at the peak (of about $16 \%$ under $0.61 \mathrm{GPa}$ and $0.05 \mathrm{~T}$ ) can also contribute to the drop in the entropy change seen in Fig. 3(b). As only $\Gamma^{4 g}$ has a symmetry-allowed ferromagnetic component [Fig. 1(b)], the reduced magnetization under pressure likely results from a variation of the $\Gamma^{4 g}: \Gamma^{5 g}$ ratio in this transitional region. However, a detailed understanding of the magnetic structure requires neutrondiffraction studies under pressure.

To calculate pressure-driven isothermal changes in entropy $\Delta S$ and pressure-driven adiabatic changes in temperature $\Delta T$, we use the total entropy curves $S-S_{210 \mathrm{~K}}$ [Fig. 3(c)] measured under various applied pressures. The isothermal entropy changes [Fig. 3(d)] are calculated upon the release of pressure to atmospheric. The peak value of $|\Delta S|$ increases to a value of approximately $35 \mathrm{~J} \mathrm{~K}^{-1} \mathrm{~kg}^{-1}$ by removing $0.28 \mathrm{GPa}$ and subsequently saturates upon removing pressure from higher values, whereas the temperature window at which the values of $|\Delta S|$ are large increases continuously with removal of higher pressures. This maximum value of $|\Delta S|$ is approximately 1.6 times larger than $\mathrm{Mn}_{3} \mathrm{GaN}$, in approximate agreement with the relative FOM values. These entropy change values are comparable to those observed in the best-performing barocaloric materials (Table I). Moreover, the high density of $\mathrm{Mn}_{3} A \mathrm{~N}$ compared to other barocaloric materials means that when measured per unit volume, $\mathrm{Mn}_{3} \mathrm{NiN}$ is outperformed only by two materials, $\mathrm{AgI}$ and $\mathrm{MnCoGe}_{0.99} \mathrm{In}_{0.01}$. Considering now $\Delta T$, both the peak value and temperature range for which $\Delta T>0$ continuously increase upon removal of the largest applied pressure of $0.56 \mathrm{GPa}$. The largest peak value of $\Delta T$ is $5.8 \mathrm{~K}$; therefore, the available adiabatic temperature change for a given pressure release $[(\Delta T) /(d p)]$ is $10.4 \mathrm{~K} \mathrm{GPa}^{-1}$. However, these values are estimated from quasidirect measurements and direct measurement of the adiabatic temperature change is required. Note that in order to drive these BCEs in a reversible manner, pressures larger than $0.56 \mathrm{GPa}$ are required.

\section{THEORETICAL MODELING}

The lack of scaling between $\Delta \omega_{T_{N}}$ and $\left|\left[\left(d T_{N}\right) /(d p)\right]\right|$ suggests properties beyond magnetovolume coupling contribute to $|\Delta S|$. To further understand the giant barocaloric entropy changes in $\mathrm{Mn}_{3} \mathrm{NiN}$, we carry out an analysis of our experimental data based on a description of the Gibbs free energy $G$. We design a simple generic form of $G$ drawing from our previous detailed $a b$ initio density functional theory-based disordered local moment theory modeling of the complex magnetic phase diagrams of the heavy rare-earth metals [30], transition-metal alloys such as FeRh [21], and pertinent to this work $\mathrm{Mn}_{3} \mathrm{GaN}$ [18]. Using this model, full details of which are in the Supplemental Material [25], we are able to write $G$ as

$$
\begin{aligned}
G= & -a_{2}\left(M_{1}^{2}+M_{2}^{2}+M_{3}^{2}\right)-a_{4}\left(M_{1}^{4}+M_{2}^{4}+M_{3}^{4}\right) \\
& -c_{\mathrm{MV}} \Delta \omega\left(M_{1}^{2}+M_{2}^{2}+M_{3}^{2}\right) \\
& +\frac{1}{2} \Omega \gamma \Delta \omega^{2}+\Omega \Delta \omega p-T S_{\mathrm{tot}},
\end{aligned}
$$

where the last term is the total entropy composed by the entropy contributions from the three sublattices $S_{\text {tot }}=S_{1}+S_{2}+S_{3}, M_{i}$ are the magnetic order parameters associated with each site $i, c_{\mathrm{MV}}$ is the magnetovolume coupling constant, and $a_{2}$ and $a_{4}$ are constants describing pairwise and the lowest possible order of multisite interactions connecting the magnetic order parameters, respectively. Note that we also add a simple elastic term proportional to the inverse of the compressibility $\gamma$ (with a value around approximately $130 \mathrm{GPa}$ for the Mn-based antiperovskites $[19,27])$ and the effect of an external hydrostatic pressure $p$. The quartic coefficients describe the effect of magnetic ordering growth on the underlying itinerant electronic structure and how the consequent changes of the electronic structure when spin polarized can affect and qualitatively alter the interactions between the local moments.

By minimizing Eq. (1) with respect to the secondary order parameter $\Delta \omega$, we obtain

TABLE I. Pressure-driven entropy changes for selected state-of-the-art barocaloric materials, listing their transition temperatures, applied pressures, and corresponding entropy changes normalized by mass or volume. Entries for $\left(\mathrm{NH}_{4}\right)_{2} \mathrm{SO}_{4}$ and $\mathrm{AgI}$ represent reversible effects. Other entries represent irreversible effects. Entropy changes are obtained from quasidirect calorimetric methods for all entries, expect for $\mathrm{MnCoGe}_{0.99} \mathrm{In}_{0.01}$ where entropy changes are estimated from pressure-dependent neutron diffraction.

\begin{tabular}{lccccc}
\hline \hline Material & Transition temperature (K) & Applied pressure $(\mathrm{GPa})$ & $\Delta S_{\max }\left(\mathrm{J} \mathrm{K}^{-1} \mathrm{~kg}^{-1}\right)$ & $\Delta S_{\max }\left(\mathrm{J} \mathrm{K}^{-1} \mathrm{~cm}^{-3}\right)$ & Reference \\
\hline $\mathrm{MnCoGe}_{0.99} \mathrm{In}_{0.01}$ & 310 & 0.3 & 52 & 0.42 & {$[11]$} \\
$\left(\mathrm{NH}_{4}\right)_{2} \mathrm{SO}_{4}$ & 224 & 0.1 & 61 & 0.11 & {$[12]$} \\
$\left(\mathrm{NH}_{4}\right)_{2} \mathrm{SnF}_{6}$ & 110 & 0.14 & 61 & 0.18 & {$[29]$} \\
$\mathrm{AgI}$ & 420 & 0.22 & 63 & 0.36 & {$[13]$} \\
$\mathrm{Mn}_{3} \mathrm{NiN}$ & 262 & 0.28 & 35 & 0.24 & This work \\
\hline \hline
\end{tabular}




$$
\Delta \omega=\frac{1}{\gamma \Omega}\left[c_{\mathrm{MV}}\left(M_{1}^{2}+M_{2}^{2}+M_{3}^{2}\right)-\Omega p\right],
$$

which substituted into Eq. (1) gives

$$
\begin{aligned}
G= & -\left[a_{2}-\frac{c_{\mathrm{MV}}}{\gamma} p\right]\left(M_{1}^{2}+M_{2}^{2}+M_{3}^{2}\right) \\
& -a_{4}\left(M_{1}^{4}+M_{2}^{4}+M_{3}^{4}\right)-\frac{c_{\mathrm{MV}}^{2}}{2 \Omega \gamma}\left(M_{1}^{2}+M_{2}^{2}+M_{3}^{2}\right)^{2} \\
& -\frac{\Omega p^{2}}{2 \gamma}-T S_{\mathrm{tot}} .
\end{aligned}
$$

The free energy expressed above can be minimized with respect to the magnetic order parameters for given values of $T$ and $p$, and, consequently, the theoretical quantities of $T_{N}$, $\left|\left[\left(d T_{N}\right) /(d p)\right]\right|$, and $\Delta \omega_{T_{N}}$ can be suitably calculated as functions of the model parameters $a_{2}, a_{4}$, and $c_{\mathrm{MV}}$.

To conclude this section, we derive the condition for which the transition from the paramagnetic state $\left(M_{1}=M_{2}=M_{3}=0\right)$ to the triangular state $\left(M_{1}=M_{2}=\right.$ $M_{3} \neq 0$ ) changes from second order to first order. As shown by Bean and Rodbell [31], this condition follows from requiring that the fourth-order coefficient in the order parameters of $G$ is negative. To find this, we first write the entropy contributions $S_{n}$ as an expansion in terms of $M_{n}$ (see the Supplemental Material [25]),

$$
S_{n}=k_{B}\left(\log 4 \pi-\frac{3}{2} M_{n}^{2}-\frac{9}{20} M_{n}^{4}-\ldots\right) .
$$

Now, from Eqs. (3) and (4) it directly follows that the condition is

$$
a_{4}=\frac{3}{10} a_{2}-\frac{3 c_{\mathrm{MV}}^{2}}{2 \Omega \gamma}
$$

\section{DETERMINING THE MULTISITE INTERACTION STRENGTH}

By minimizing $G$ of Eq. (3) with respect to the magnetic order parameters for given values of $T$ and $p$, the theoretical quantities of $T_{N},\left|\left[\left(d T_{N}\right) /(d p)\right]\right|$, and $\Delta \omega_{T_{N}}$ can be calculated as a function of the model parameters $a_{2}, a_{4}$, and $c_{\text {MV }}$. For instance, we show in Figs. 4(a) and 4(b) how $T_{N}$ and $\left|\left[\left(d T_{N}\right) /(d p)\right]\right|$ depend on $a_{2}$ and $c_{\mathrm{MV}}$, respectively, while dependences of $a_{4}$ on (i) $c_{\mathrm{MV}}$ and $\left|\left[\left(d T_{N}\right) /(d p)\right]\right|$ and (ii) $c_{\mathrm{MV}}$ and $\Delta S$ are shown in Figs. 4(c) and 4(d), respectively. Using this model combined with our experimental data on $\mathrm{Mn}_{3} \mathrm{NiN}$ and literature data on $\mathrm{Mn}_{3} \mathrm{GaN}$ [15], the constants $a_{2}, a_{4}$, and $c_{\mathrm{MV}}$ are determined by an iterative fitting process for both systems. The final parameters are shown in Table II.

$\mathrm{Mn}_{3} \mathrm{GaN}$ has a significantly larger $c_{\mathrm{MV}}$ of $1107 \mathrm{meV}$ compared to the small value of $250 \mathrm{meV}$ for $\mathrm{Mn}_{3} \mathrm{NiN}$,
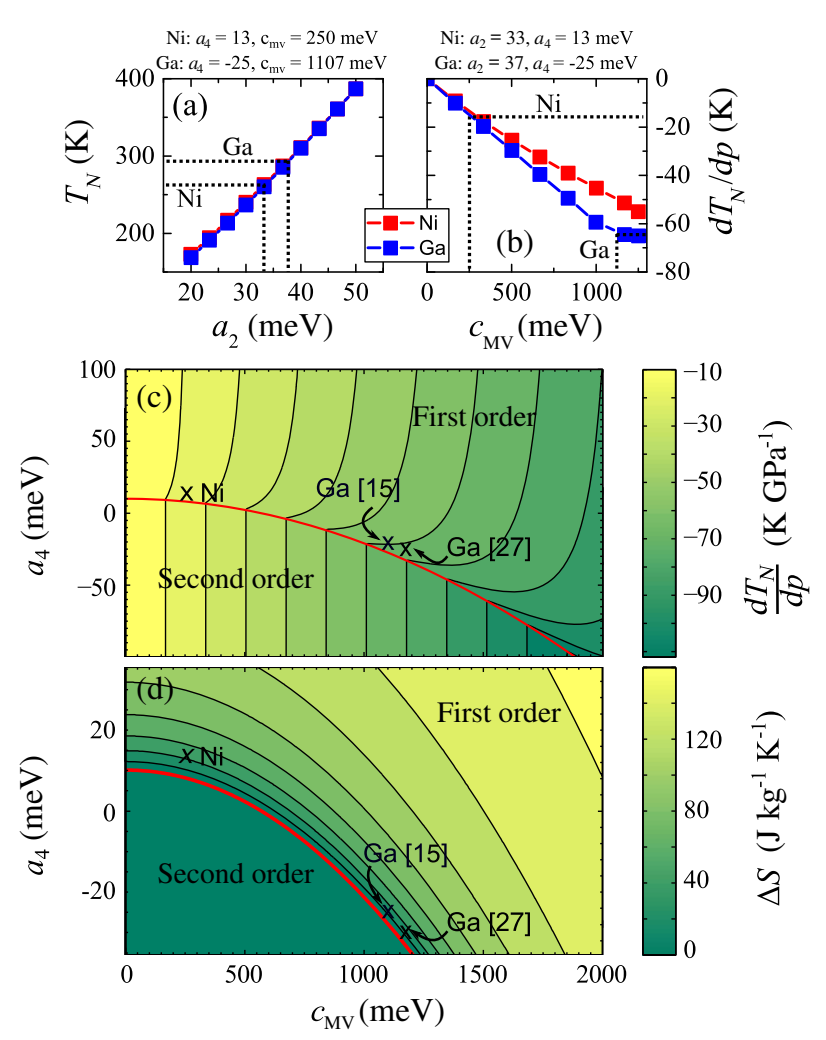

FIG. 4. The theoretical quantities (a) $T_{N}$ and (b) $\left[\left(d T_{N}\right) /(d p)\right]$ as a function of $a_{2}$ and $c_{\mathrm{MV}}$, respectively, calculated using our model described in the text. Dotted lines indicate the experimentally measured values for $A=\mathrm{Ni}$ and $\mathrm{Ga}$. The parameters used are shown above each plot. (c),(d) The dependence of (c) $\left[\left(d T_{N}\right) /(d p)\right]$ and (d) $\Delta S$ as a function of $a_{4}$ and $c_{\mathrm{MV}}$ with $a_{2}=36 \mathrm{meV}$ for both nitride systems. The plot is effectively insensitive to the $a_{2}$ value for the pertinent range $\left(33<a_{2}<\right.$ $37 \mathrm{meV}$ ). The red line indicates the critical curve separating firstand second-order behavior.

consistent with the larger volume change at the transition. We propose that the 5-times-larger ratio $\left|\left[\left(d T_{N}\right) /(d p)\right]\right|$ observed experimentally in $\mathrm{Mn}_{3} \mathrm{GaN}$ mainly originates from the larger magnetovolume coupling. Turning now to the $a_{2}$ and $a_{4}$ terms, we observe that $a_{2}$ scales mainly with $T_{N}$, while positive $a_{4}$ contributes to the first-order character of the transition [see Eq. (3)], in agreement with other models based on expanding the free energy in terms of the magnetic order parameter [31,32]. Therefore, while $\Delta \omega_{T_{N}}$ at the transition is smaller in $\mathrm{Mn}_{3} \mathrm{NiN}$ compared to $\mathrm{Mn}_{3} \mathrm{GaN}$, the entropy change can be at least as large due to the smaller but positive $a_{4}$ term preserving a substantial $\Delta \omega_{T_{N}}$ relative to the low $\left|\left[\left(d T_{N}\right) /(d p)\right]\right|$ from the small magnetovolume coupling This explanation is exemplified in Fig. 4(c), which shows the dependence of $\left|\left[\left(d T_{N}\right) /(d p)\right]\right|$ on $a_{4}$ and $c_{\mathrm{MV}}$. It is clear from this plot that $\left|\left[\left(d T_{N}\right) /(d p)\right]\right|$ scales with $c_{\mathrm{MV}}$, while increasing positive $a_{4}$ leads to more first-order behavior. Hence, we understand that $\mathrm{Ni}$ is pushed towards the region displaying first-order behavior and, therefore, larger $\Delta S$ [see Fig. 4(d)]. As the $a_{4}$ term originates from the 
TABLE II. Experimental values of $T_{N}, \Delta \omega_{T_{N}}$, and $\left[\left(d T_{N}\right) /(d p)\right]$ for $\mathrm{Mn}_{3} \mathrm{NiN}$ and $\mathrm{Mn}_{3} \mathrm{GaN}$ along with the calculated values of $c_{\mathrm{MV}}$, $a_{2}, a_{4}$, and $\Delta S$ as described in the text. The latter, $\Delta S_{\mathrm{CC}}$, is calculated from the Clausius-Clapeyron equation but in excellent agreement with that determined from calorimetry. The maximum magnetic entropy available for an $S=\frac{5}{2}$ Mn ion, commonly given by $\Delta S=$ $k_{B} \ln (2 S+1)$ in $\mathrm{Mn}_{3} \mathrm{NiN}$ and $\mathrm{Mn}_{3} \mathrm{GaN}$ is 188.2 and $179.8 \mathrm{~J} \mathrm{~kg}^{-1} \mathrm{~K}^{-1}$, respectively. As such, the $\mathrm{Mn}_{3} A \mathrm{~N}$ family has the potential to realize much larger entropy changes.

\begin{tabular}{lccccccc}
\hline \hline & $T_{N}(\mathrm{~K})$ & $\Delta \omega_{T_{N}}(\%)$ & {$\left[\left(d T_{N}\right) /(d p)\right]\left(\mathrm{K} \mathrm{GPa}^{-1}\right)$} & $c_{\mathrm{MV}}(\mathrm{meV})$ & $a_{2}(\mathrm{meV})$ & $a_{4}(\mathrm{meV})$ & $\Delta S_{\mathrm{CC}}\left(\mathrm{J} \mathrm{K}^{-1} \mathrm{~kg}^{-1}\right)$ \\
\hline $\mathrm{Mn}_{3} \mathrm{NiN}$ & 262 & 0.4 & 13.5 & 250 & 33 & 13 & 47 \\
$\mathrm{Mn}_{3} \mathrm{GaN}$ & $290[15]$ & $1.0[15]$ & $65[15]$ & 1107 & 37 & -25 & 22 \\
\hline \hline
\end{tabular}

multisite interactions among the Mn local moments and the itinerant electron spin, it appears consistent that the larger $\mathrm{BCE}$ in $\mathrm{Mn}_{3} \mathrm{NiN}$ relative to $\mathrm{Mn}_{3} \mathrm{GaN}$ is due to these multisite terms providing an additional contribution that strongly favors the triangular state. This information provides a useful handle on the barocaloric properties of $\mathrm{Mn}_{3} A \mathrm{~N}$ and stimulates research on those with small magnetovolume coupling that may have been overlooked. For instance, $A=\mathrm{Co}, \mathrm{Pd}$, and $\mathrm{Rh}$ all have significantly lower magnetovolume effects than $A=\mathrm{Ni}$ [27], but their magnetism may have a contribution from the multisite exchange that enhances the BCE based on their potential to hybridize with the Mn $d$ band (as is the case for Ni) [19]. Moreover, it is possible to tailor quaternary compounds that offer large BCE at room temperature [27].

\section{CONCLUSION AND OUTLOOK}

In conclusion, we measure the barocaloric properties of the geometrically frustrated antiferromagnet $\mathrm{Mn}_{3} \mathrm{NiN}$, a member of the Mn-based antiperovskite family $\mathrm{Mn}_{3} A \mathrm{~N}$. This material displays large pressure-driven isothermal entropy changes and adiabatic temperature changes of $\Delta S=35 \mathrm{~J} \mathrm{~K}^{-1} \mathrm{~kg}^{-1} \quad(p=0.28 \mathrm{GPa})$ and $\Delta T=5.8 \mathrm{~K}$ $(p=0.56 \mathrm{GPa})$ near the paramagnetic to antiferromagnetic transition. The isothermal entropy change is larger than the closely related antiperovskite $\mathrm{Mn}_{3} \mathrm{GaN}$ by a factor of 1.6 and within a factor of 2 of the largest entropy changes reported in the literature once normalization by density is taken into account. Considering that the magnetovolume coupling is a factor of 5 smaller in $\mathrm{Mn}_{3} \mathrm{NiN}$ compared to $A=\mathrm{Ga}$, the results are in stark contrast to the anticipated trend that larger magnetovolume coupling naturally leads to a larger BCE. Our theoretical insights reveal that their properties are strongly linked to a combination of electronic spin effects and lattice changes. The interactions between the local moments associated with the magnetic Mn sites depend on the overall magnetic order described by the multisite interactions. Their positive value, which is set by our experimental data, acts to enhance the first-order nature of the transition in $\mathrm{Mn}_{3} \mathrm{NiN}$, and, consequently, the material retains a large volume change despite the relatively small magnetovolume coupling. This improved understanding can be used to tune the $\mathrm{BCE}$ in $\mathrm{Mn}_{3} A \mathrm{~N}$ materials and highlights the potential of discovering new materials with enhanced properties in this broad and chemically flexible family as well as frustrated magnets in general.

\section{DATA AVAILABILITY}

All relevant data are presented via this publication and Supplemental Material [25].

\section{ACKNOWLEDGMENTS}

We thank A. S. Wills for help with sample preparation. This work is supported by EPSRC (UK) Grants No. EP/P511109/ 1, No. EP/P030548/1, No. EP/J006750/1, and No. EP/ M028941/1, MINECO (Spain) Projects No. FIS201454734-P, No. 2017SGR-42 (Catalan government), and ERC Starting Grant No. 680032. X. M. is grateful for support from the Royal Society.

[1] L. Mañosa and A. Planes, Materials with Giant Mechanocaloric Effects: Cooling by Strength, Adv. Mater. 29, 1603607 (2017).

[2] N. H. Dung, Z. Q. Ou, L. Caron, L. Zhang, D. T. C. Thanh, G. A. De Wijs, R. A. De Groot, K. H. J. Buschow, and E. Brück, Mixed Magnetism for Refrigeration and Energy Conversion, Adv. Energy Mater. 1, 1215 (2011).

[3] X. Moya, S. Kar-Narayan, and N. D. Mathur, Caloric Materials near Ferroic Phase Transitions, Nat. Mater. 13, 439 (2014).

[4] N. K. Singh, K. G. Suresh, A. K. Nigam, S. K. Malik, A. A. Coelho, and S. Gama, Itinerant Electron Metamagnetism and Magnetocaloric Effect in $\mathrm{RCo}_{2}$-based Laves Phase Compounds, J. Magn. Magn. Mater. 317, 68 (2007).

[5] A. Fujita and K. Fukamichi, Giant Volume Magnetostriction due to the Itinerant Electron Metamagnetic Transition in $\mathrm{La}(\mathrm{Fe}-\mathrm{Si})_{13}$ Compounds, IEEE Trans. Magn. 35, 3796 (1999).

[6] M. E. Gruner, W. Keune, B. R. Cuenya, C. Weis, J. Landers, S. I. Makarov, D. Klar, M. Y. Hu, E. E. Alp, J. Zhao, M. Krautz, O. Gutfleisch, and H. Wende, Element-Resolved Thermodynamics of Magnetocaloric $\mathrm{LaFe}_{13-x} \mathrm{Si}_{x}$, Phys. Rev. Lett. 114, 057202 (2015).

[7] S. Yuce, M. Barrio, B. Emre, E. Stern-Taulats, A. Planes, J.-L. Tamarit, Y. Mudryk, K. A. Gschneidner, V. K. Pecharsky, and L. Mañosa, Barocaloric Effect in the Magnetocaloric Prototype $\mathrm{Gd}_{5} \mathrm{Si}_{2} \mathrm{Ge}_{2}$, Appl. Phys. Lett. 101, 071906 (2012).

[8] L. Mañosa, D. González-Alonso, A. Planes, E. Bonnot, M. Barrio, J.-L. Tamarit, S. Aksoy, and M. Acet, Giant 
Solid-State Barocaloric Effect in the NiMnIn Magnetic Shape-Memory Alloy, Nat. Mater. 9, 478 (2010).

[9] L. Mañosa, D. González-Alonso, A. Planes, M. Barrio, J.-L. Tamarit, I. S. Titov, M. Acet, A. Bhattacharyya, and S. Majumdar, Inverse Barocaloric Effect in the Giant Magnetocaloric LaFeSiCo Compound, Nat. Commun. 2, 595 (2011).

[10] E. Stern-Taulats, A. Planes, P. Lloveras, M. Barrio, J.-L. Tamarit, S. Pramanick, S. Majumdar, C. Frontera, and L. Mañosa, Barocaloric and Magnetocaloric Effects in $\mathrm{Fe}_{49} \mathrm{Rh}_{51}$, Phys. Rev. B 89, 214105 (2014).

[11] R.-R. Wu et al., Giant Barocaloric Effect in Hexagonal $\mathrm{Ni}_{2}$ In-type Mn-Co-Ge-In Compounds around Room Temperature, Sci. Rep. 5, 18027 (2015).

[12] P. Lloveras, E. Stern-Taulats, M. Barrio, J.-L. Tamarit, S. Crossley, W. Li, V. Pomjakushin, A. Planes, L. Mañosa, N. D. Mathur, and X. Moya, Giant Barocaloric Effects at Low Pressure in Ferrielectric Ammonium Sulphate, Nat. Commun. 6, 8801 (2015).

[13] A. Aznar, P. Lloveras, M. Romanini, M. Barrio, J.-L. Tamarit, C. Cazorla, D. Errandonea, N. D. Mathur, A. Planes, X. Moya, and L. Mañosa, Giant Barocaloric Effects over a Wide Temperature Range in Superionic Conductor AgI, Nat. Commun. 8, 1851 (2017).

[14] J. M. Bermudez-Garcia, M. Sanchez-Andujar, S. CastroGarcia, J. Lopez-Beceiro, R. Artiaga, and M. A. SenarisRodriguez, Giant Barocaloric Effect in the Ferroic Organic-Inorganic Hybrid $[\mathrm{TPrA}]\left[\mathrm{Mn}(\mathrm{dca})_{3}\right]$ Perovskite under Easily Accessible Pressures, Nat. Commun. 8, 15715 (2017).

[15] D. Matsunami, A. Fujita, K. Takenaka, and M. Kano, Giant Barocaloric Effect Enhanced by the Frustration of the Antiferromagnetic Phase in $\mathrm{Mn}_{3} \mathrm{GaN}$, Nat. Mater. 14, 73 (2015).

[16] E. O. Chi, W. S. Kim, and N. H. Hur, Nearly Zero Temperature Coefficient of Resistivity in Antiperovskite Compound $\mathrm{CuNMn}_{3}$, Solid State Commun. 120, 307 (2001).

[17] S. Deng, Y. Sun, L. Wang, Z. Shi, H. Wu, Q. Huang, J. Yan, K. Shi, P. Hu, A. Zaoui, and C. Wang, Frustrated Triangular Magnetic Structures of $\mathrm{Mn}_{3} \mathrm{ZnN}$ : Applications in Thermal Expansion, J. Phys. Chem. C 119, 24983 (2015).

[18] J. Zemen, E. Mendive-Tapia, Z. Gercsi, R. Banerjee, J. B. Staunton, and K. G. Sandeman, Frustrated Magnetism and Caloric Effects in Mn-Based Antiperovskite Nitrides: Ab Initio Theory, Phys. Rev. B 95, 184438 (2017).

[19] J. Zemen, Z. Gercsi, and K. G. Sandeman, Piezomagnetic Effect as a Counterpart of Negative Thermal Expansion in Magnetically Frustrated Mn-based Antiperovskite Nitrides, Phys. Rev. B 96, 024451 (2017).
[20] D. Boldrin, A. P. Mihai, B. Zou, J. Zemen, R. Thompson, E. Ware, B. V. Neamtu, L. Ghivelder, B. Esser, D. W. McComb, P. Petrov, and L. F. Cohen, Giant Piezomagnetism in $\mathrm{Mn}_{3} \mathrm{NiN}$, ACS Appl. Mater. Interfaces 10, 18863 (2018).

[21] J. B. Staunton, R. Banerjee, M. dos Santos Dias, A. Deak, and L. Szunyogh, Fluctuating Local Moments, Itinerant Electrons, and the Magnetocaloric Effect: Compositional Hypersensitivity of FeRh, Phys. Rev. B 89, 054427 (2014).

[22] S. Nakatsuji, N. Kiyohara, and T. Higo, Large Anomalous Hall Effect in a Non-Collinear Antiferromagnet at Room Temperature, Nature (London) 527, 212 (2015).

[23] D. Boldrin and L.F. Cohen, The Role of Competing Magnetic Interactions on the Abnormal Expansion Properties in Manganese Antiperovskites, $\mathrm{Mn}_{3+x} A_{1 x} \mathrm{~N}(A=\mathrm{Ni}$, Sn), J. Alloys Compd. 699, 887 (2017).

[24] D. Boldrin, Z. Gercsi, K. G. Sandeman, T. Hansen, and L. F. Cohen, Engineering Magnetic Competition in $\mathrm{Mn}_{3+x} \mathrm{~T}_{1-x} \mathrm{~N}$, Inst. Laue-Langevin, doi: 10.5291/ILL-DATA.5-31-2465.

[25] See Supplemental Material at http://link.aps.org/ supplemental/10.1103/PhysRevX.8.041035 for a description of the experimental details, the entropy calculations and the theoretical modeling.

[26] D. Fruchart, E. F. Bertaut, R. Madar, G. Lorthioir, and R. Fruchart, Structure Magnétique et Rotation de Spin de $\mathrm{Mn}_{3} \mathrm{NiN}$, Solid State Commun. 9, 1793 (1971).

[27] K. Takenaka, M. Ichigo, T. Hamada, A. Ozawa, T. Shibayama, T. Inagaki, and K. Asano, Magnetovolume Effects in Manganese Nitrides with Antiperovskite Structure, Sci. Technol. Adv. Mater. 15, 015009 (2014).

[28] M. Wu, C. Wang, Y. Sun, L. Chu, J. Yan, D. Chen, Q. Huang, and J.W. Lynn, Magnetic Structure and Lattice Contraction in $\mathrm{Mn}_{3} \mathrm{NiN}$, J. Appl. Phys. 114, 123902 (2013).

[29] I. N. Flerov, A. V. Kartashev, M. V. Gorev, E. V. Bogdanov, S. V. Mel'Nikova, M. S. Molokeev, E. I. Pogoreltsev, and N. M. Laptash, Thermal, Structural, Optical, Dielectric and Barocaloric Properties at Ferroelastic Phase Transition in Trigonal $\left(\mathrm{NH}_{4}\right)_{2} \mathrm{SnF}_{6}$ : A New Look at the Old Compound, J. Fluorine Chem. 183, 1 (2016).

[30] E. Mendive-Tapia and J. B. Staunton, Theory of Magnetic Ordering in the Heavy Rare Earths: Ab Initio Electronic Origin of Pair-and Four-Spin Interactions, Phys. Rev. Lett. 118, 197202 (2017).

[31] C. P. Bean and D. S. Rodbell, Magnetic Disorder at a First Order Phase Transformation, Phys. Rev. 126, 104 (1962).

[32] E. Mendive-Tapia and T. Castán, Magnetocaloric and Barocaloric Responses in Magnetovolumic Systems, Phys. Rev. B 91, 224421 (2015). 